\title{
Hybrid Electric Vehicle Two-step Fuel Efficiency Optimization with Decoupled Energy Management and Speed Control
}

\author{
Boli Chen, Simos A. Evangelou and Roberto Lot
}

\begin{abstract}
Hybrid electric vehicles (HEVs) offer an effective solution for emissions reduction and fuel energy savings. The pursuit of further improvements in their energy efficiency has led to the two fundamental optimization challenges of vehicle speed and powertrain energy management (EM), which are inherently coupled. This paper examines the vehicle speed and powertrain EM co-optimization problem for fuel economy for a series HEV following a prescribed route with expected traveling time. In order to overcome the computational burden of a large scale optimal control problem (OCP), this work presents a novel twostep optimal control strategy that suitably separates the cooptimization problem on the basis of involving the characteristics of the HEV powertrain power split and losses in the speed optimization step without an explicit use of a powertrain model. A benchmark method that simultaneously solves the optimal driving speed and the energy source power split is introduced, which is used to show the solution quality of the proposed approach. It is illustrated that the proposed method yields a driving speed solution close to the benchmark method, and additionally it outperforms the benchmark fuel economy, with much higher computational efficiency. The simplicity and effectiveness of the proposed two-step approach make it a practical and implementable EM control strategy.
\end{abstract}

\begin{tabular}{ll} 
& \multicolumn{1}{c}{ ABBREVIATIONS } \\
CS & Charge-Sustaining \\
ECMS & Equivalent Consumption Minimization Strategy \\
EM & Energy Management \\
HEV & Hybrid Electric Vehicle \\
ICE & Internal Combustion Engine \\
NLP & Nonlinear Programming \\
OCP & Optimal Control Problem \\
PL & Propulsion Load \\
PMS & Permanent Magnet Synchronous (Machine) \\
PS & Primary Source of energy \\
SOC & State of Charge \\
SS & Secondary Source of energy
\end{tabular}

\section{INTRODUCTION}

Concerns about climate change and the increasing global energy consumption have led to the electrification of ground

Copyright (c) 2015 IEEE. Personal use of this material is permitted. However, permission to use this material for any other purposes must be obtained from the IEEE by sending a request to pubs-permissions@ieee.org.

B. Chen is with the Dept. of Electronic and Electrical Engineering at University College London, UK (boli . chen @ucl. ac.uk).

$\mathrm{S}$. A. Evangelou is with the Dept. of Electrical and Electronic Engineering at Imperial College London, UK ( . evangelou@imperial.ac.uk) .

R. Lot is with the Dept. of Engineering and the Environment at the University of Southampton, UK (Roberto. Lotesoton.ac.uk).

This research was supported by the EPSRC Grant EP/N022262/1. vehicles, which have great potential to reduce greenhouse gas emissions and to save fossil fuels. As with the conventional internal combustion engine (ICE) vehicles, the energy efficiency of a hybrid electric vehicle (HEV) can be enhanced in two aspects: a) "vehicle-to-miles", and b) "tank-to-vehicle" [1]. The first step a) represents the energy conversion from mechanical energy received from the powertrain system to the kinetic energy for the displacement, while in the second energy conversion step b) the fuel energy is transformed to the mechanical energy for propulsion via the hybrid powertrain. In the literature on HEV energy efficiency optimization, a) is primarily addressed by optimizing driving speed, which also plays an important role in eco-driving training schemes. On the other hand, the approach of improving "tank-to-vehicle" efficiency is usually referred to as an energy management (EM) strategy that aims at minimizing overall fuel consumption by a suitable energy source power-split, while following a predefined driving cycle.

The energy-aware driving style has been thoroughly investigated in the last two decades for conventional vehicles. It has been shown using the theory of optimal control in [2], [3] and experimentally in [4] that when the traffic and road elevation is not considered, pulse and glide (PnG) operation is fuel efficient. Such operation includes a rapid acceleration at the beginning until the vehicle reaches its maximum speed, followed by a period of coasting to a low speed. The operation of heavy-duty vehicles diverts from PnG, since their optimal driving speed is strongly influenced by the road gradient. In [5], look-ahead controllers are proposed for optimizing the speed of heavy trucks in a highway based on trip information, which entails the road slope ahead of the vehicle. In contrast with the highway condition, the urban driving scenario is usually subject to the infrastructure constraints (e.g., traffic lights), which increase the problem complexity. Driving speed optimization in such circumstances has been addressed in [6] by a predictive control method, while the influence of other perturbations, including engine saturation, headwind and traffic flow speed is systematically investigated in [7]. Increased research attentions have recently been paid also to the speed control of connected and automated vehicles, so as to find the energy-efficient and safe cruise speed according to the traffic and geographical information of the road networks [8], [9], [10], [11]. Furthermore, the optimal speed of a heavyduty vehicle platoon is investigated in [12], where a twolayer controller, formed by dynamic programming (DP) and model predictive control (MPC) is proposed to safely and fuel- 
efficiently coordinate the vehicles in the platoon. Extending the conventional eco-driving strategy to full electric vehicles (BEVs) with a single electric energy source is straightforward and it is investigated in [13] by an optimal control approach. The optimal solution in this case involves a smoother acceleration and lower peak speed as compared to a PnG profile. Despite this, there is still a lack of investigation on the energyefficient driving of HEVs, which are characterized by a higher level of complexity due to the interaction between vehicle driving speed and hybrid powertrain EM control.

There is a rich literature in the powertrain EM control design, which is one of the main challenges for modern HEVs. A comprehensive overview of existing EM control techniques can be found in [14], [15], [16]. According to the fundamental design principles, EM strategies are classified into two main categories: optimization-based and rule-based approaches. The former group encompasses numerous algorithms, such as DP [17], [18], equivalent consumption minimization strategy (ECMS) [19], [20], MPC [21], [22], [23] and emerging approaches based on machine learning and neural networks [24], [25], [26], [27]. Among these, DP is able to find global optimal solutions. However, it is not suitable for practical use as the computational burden increases exponentially with the model complexity [28]. ECMS is a commonly used costeffective alternative, which is derived from the Pontryagin's Minimum Principle (PMP) optimality condition. The main drawback of the ECMS is that the tuning of the equivalence factors is not straightforward, due to their high sensitivity to characteristics of the drive cycle [29]. On the other hand, the rule-based techniques define the vehicle operating modes by Boolean or fuzzy rules, which are easy to implement and understand, such as thermostat control strategy and power follower control strategy. Nonetheless, conventional rule-based methods are very inefficient and lack of charge sustaining (CS) guarantees. The rule-based Exclusive operation strategy (XOS) [30] and Optimal primary source strategy (OPSS) [31] have recently been introduced for series HEVs. The XOS and OPSS have some of their features inspired by the ECMS and both strategies ensure CS operation by a so called thresholdchanging mechanism that is defined by battery state-of-charge (SOC) dependent power thresholds. Although XOS and OPSS can yield near-optimal solutions for particular drive cycles, they still fall behind by some margin from optimization-based benchmarks.

One of the main drawbacks of most of the EM control methodologies in the literature is that they require a priori the knowledge of the entire drive cycle, which is practically unavailable. In this context, it is more appropriate to define the driving mission in terms of a path (referred to as a "free driving" problem) or the preceding vehicle speed (referred to as a "car following" problem). The resulting optimal control problem (OCP) may be addressed by solving individual speed and EM optimization problems, one for each aspect, in steps and combined a posteriori. However, the solution must be suboptimal as the conventional speed optimization methods do not incorporate the characteristics of the hybrid powertrain and power sharing strategies. To overcome the disadvantages of such a two-step optimization mechanism, simultaneous optimization of both driving speed and EM has also been suggested (see for example [32], [33], [34], [35]), which is expected to achieve better optimal solutions than the two-step counterpart. However, the computation complexity is increased when both aspects are simultaneously solved. The prior work presented by the present authors [36] has examined the benefit and challenges of the joint optimization strategy on the basis of a series HEV architecture. The joint optimization solution is benchmarked against a two-step algorithm with an introduced speed optimization algorithm that can emulate the energy recovery phenomenon of an $\mathrm{HEV}$, and consequently improves the optimality of the fuel economy solution of the associated two-step scheme. Nonetheless, the fuel economy solution of the two-step approach in [36] still lags the joint optimization result by a noticeable margin.

This paper proposes a computationally efficient two-step optimization algorithm that is formed by a novel driving speed optimization strategy, which is the core contribution of the paper, and a standard optimal EM control strategy. The proposed speed optimization strategy outperforms the algorithm presented in [36] by suitably integrating essential characteristics of the powertrain losses and optimal EM solutions without embedding the explicit powertrain model. The resulting optimal speed profile can be very close to that of the joint optimization, and when combined with the EM strategy, the associated fuel economy solution can be nearoptimal as compared to the joint optimization benchmark, with much lower computational complexity. The focus of the paper is on the series HEV architecture that is a common arrangement for modern HEVs and involves a number of products in the market, such as the Nissan Note e-power and a variety of extended-range electric vehicles. Nevertheless, the ideas that will be presented also have relevance to other HEV architectures.

The paper is organized as follows. The models of the vehicle and its powertrain are described in Section II, where the benchmark strategy with joint consideration of optimal vehicle speed and energy management is also introduced. Section III introduces the proposed two-step control strategy that is based on a separate OCP formulation for each of the driving speed and power split. Section IV provides two case studies in which the solutions of both strategies are compared. Finally, Section V comes to a conclusion on the basis of the presented work.

\section{Model DESCRIPTION AND PROBLEM STATEMENT}

In this section, the problem of optimal energy management including speed control is formulated for an HEV with a series configuration, as shown in Fig. 1. The proposed methodology aims to solve a "free driving" problem, where the vehicle is requested to complete a mission with minimum fuel usage. In particular, the mission is defined in terms of the route and the expected traveling time. Road traffic is not considered in this work, thus the vehicle speed is not limited by the behavior of other road users or infrastructure (e.g., traffic lights). However, it is straightforward to take the traffic flow into account by formulating a "car following" problem, where the mission is 


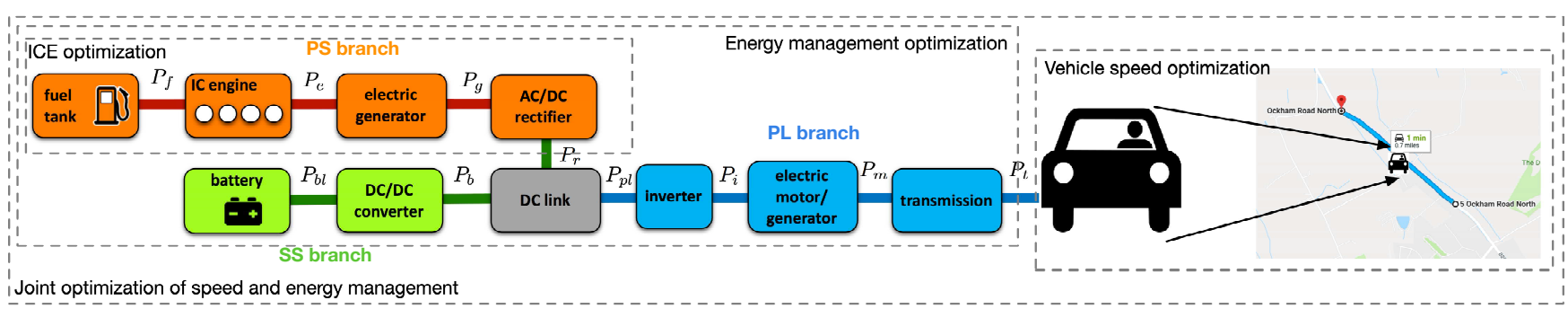

Fig. 1. Block diagram of the series HEV powertrain used in this work. The powertrain comprises the primary (PS) and secondary (SS) source branches, and the propulsion load (PL) branch, shown respectively in brown, green and blue colors.

determined by the speed of the preceding vehicle rather than the path, and the headway distance between the two vehicles is subject to prescribed constraints.

\section{A. Vehicle Model}

With reference to Fig. 1, the series HEV powertrain is formed by three branches, the primary source (PS) branch, the secondary source (SS) branch and the propulsion load (PL) branch, which are joined electrically at the DC-link. The PS consists of an engine-generator unit (EGU) and a threephase rectifier to convert chemical fuel energy to mechanical propulsion energy, and then to electric energy. The EGU contains an internal combustion engine (ICE) and a permanent magnet synchronous (PMS) generator, which are mechanically coupled. The secondary source (SS) branch contains a lithiumion battery and a bi-directional DC-DC converter. The PS and SS branches power outputs are merged at the DC link. Then, the total power is delivered to the wheels of the vehicle via the PL branch that combines an inverter, a PMS motor/generator and the transmission system. The SS allows energy recovery either by the PS or by regenerative braking, which conveys the kinetic braking energy from the wheels up to the SS by the motor behaving as a generator. In addition, mechanical brakes are applied directly to the wheels for further deceleration, with the corresponding power converted into heat dissipation.

1) Primary source: The dynamics of the ICE (e.g., engine speed, air mass flow rate) are much faster than the dynamics of interest in the present work, such as powertrain energy flow and battery SOC variations. Hence, a characterization of the average engine efficiency at steady-state operating conditions is adequate for the present purposes. Figure 2 shows the engine map utilized in this work, which is for the 1.8L Audi 5-Cylinder Turbo Diesel Engine taken from the Advanced Vehicle Simulator (ADVISOR) [37]. The generator efficiency map is also shown in Fig. 2, which is the same for the electric motor used in the PL branch. The map is calculated analytically from the mathematical model given in [32], in which the model parameters are selected to provide a realistic representation of the machine efficiency as compared to experimental data available in the literature. Finally, the rectifier is modeled as a constant efficiency factor, denoted by $\eta_{r}$. In this context, the engine branch is reasonably modeled by a steady state efficiency map, which is simply obtained as the product of individual component efficiencies, as illustrated in Fig. 3.

Since the EGU is mechanically decoupled from the driving wheels, the EGU is allowed to be controlled independently of
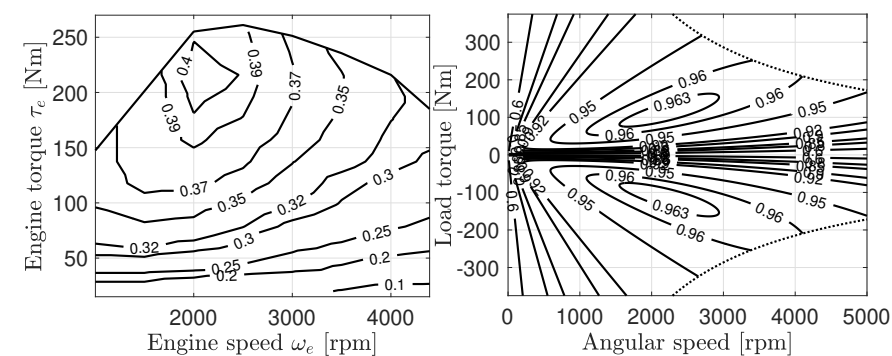

Fig. 2. Left: Map of ICE efficiency in terms of engine speed and torque. Right: Efficiency of the reversible PMS machine (generator = positive torque, motor $=$ negative torque).

the vehicle speed. By operating the engine branch along the locus of most efficient power-speed operating points (which are highlighted in the branch efficiency map in Fig. 3), the nonlinear relationship between the fuel chemical power, $P_{f}$ and the PS output power, $P_{r}$ can be accurately fitted by a linear function, as shown in Fig. 3. Therefore, the fuel mass

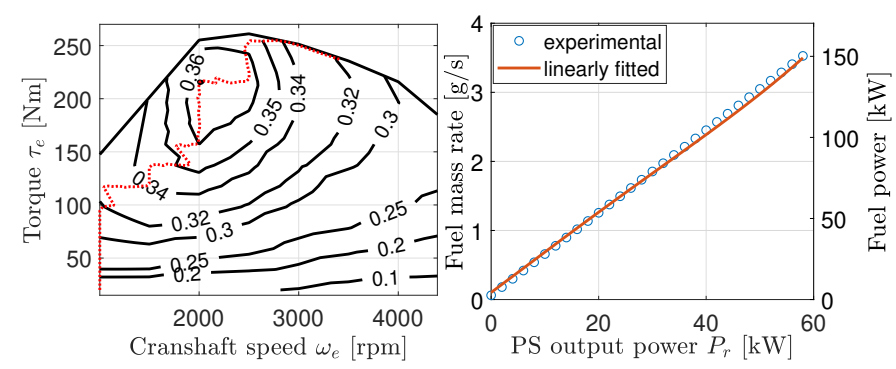

Fig. 3. Left: map of overall efficiency of the PS branch. The torque-speed operating points for maximum PS branch efficiency at different output power values are shown by a dashed curve. Right: Fuel mass rate with PS power when the most efficient torque-speed operating point is followed at each power value.

dynamic equation is given by

$$
\frac{d}{d t} m_{f}=q_{f 0}+\alpha_{f} P_{r}
$$

where $q_{f 0}$ acts as the idling fuel mass rate, and $\alpha_{f}$ is the coefficient of power transformation, obtained by using simple linear regression methods.

2) Secondary source: The dynamic of the battery is governed by:

$$
\frac{d}{d t} \mathrm{SOC}=\frac{i_{b}}{Q_{\max }} .
$$

where $i_{b}$ denotes the battery current, assumed positive during the discharge phase. For a CS battery management with low SOC variation (compatible with the usual operational strategy 
of non-plug-in HEVs), it is acceptable to assume a constant open circuit voltage, $V_{o c}$, rather than utilizing a nonlinear mapping between SOC and the open circuit voltage (as it is known in the literature). Based on the internal resistance electrical model [38], the battery closed circuit voltage is approximated by $V_{b}=V_{o c}-R_{b} i_{b}$. The battery voltage is amplified by the DC/DC converter that is modeled as a constant efficiency term $\eta_{d c}$. Therefore, the bi-directional power conversion of the SS branch is described by:

$$
P_{b}=\eta_{d c}^{\operatorname{sign}\left(P_{b}\right)} P_{b l}=\eta_{d c}^{\operatorname{sign}\left(P_{b}\right)} i_{b} V_{b},
$$

where $P_{b l}=i_{b} V_{b}$ represents the battery output power and $P_{b}$ is the SS branch output power. Equation (3) is solved with respect to $i_{b}$ and the solution

$$
i_{b}=\frac{V_{o c}-\sqrt{V_{o c}^{2}-4 P_{b} R_{b} / \eta_{d c}^{\operatorname{sign}\left(P_{b}\right)}}}{2 R_{b}},
$$

is applied to reformulate (2), leading to the differential equation of SOC with respect to $P_{b}$ :

$$
\frac{d}{d t} \mathrm{SOC}=\frac{-V_{o c}+\sqrt{V_{o c}^{2}-4 P_{b} R_{b} / \eta_{d c}^{\operatorname{sign}\left(P_{b}\right)}}}{2 R_{b} Q_{\max }} .
$$

3) Propulsion load: Similarly to the AC-DC rectifier, the DC-AC inverter is also modeled as a constant efficiency factor $\eta_{i}$. The power flow through the DC-link and inverter is described by:

$$
P_{i}=\eta_{i}^{\operatorname{sign}\left(P_{p l}\right)} P_{p l}
$$

where $P_{p l}$ is the demanded PL branch power.

The PMS motor/generator offers bi-directional energy conversion according to:

$$
P_{m}=\left(\eta_{m}\left(\tau_{l m}, \omega_{m}\right)\right)^{\operatorname{sign}\left(-P_{i}\right)} P_{i},
$$

such that when it works as a motor, the electric power $P_{i}$ is converted into mechanical power $P_{m}$ for propulsion. Conversely, $P_{m}$ is transformed into $P_{i}$ when it works as a generator. The efficiency $\eta_{m}$ of the PMS motor/generator varies with load torque $\tau_{l m}\left(=-P_{m} / \omega_{m}\right)$ and angular speed $\omega_{m}$ operating points, and it is shown in Fig. 2 .

The motor drives the wheels via the transmission system characterized by a fixed ratio $g_{t}$, such that

$$
\omega_{m}=g_{t} v
$$

in which $v$ is the vehicle longitudinal speed. The bi-directional power flow through the transmission is modeled by:

$$
P_{t}=\eta_{t}^{\operatorname{sign}\left(P_{m}\right)} P_{m}
$$

with $\eta_{t}$ a constant efficiency factor. Combing all three components of the PL branch, the overall PL power flow is:

$$
P_{p l}=\eta_{p l}^{-\operatorname{sign}\left(P_{t}\right)} P_{t}
$$

in which the PL efficiency is given by $\eta_{p l}=\eta_{i} \eta_{m} \eta_{t}$.

Let $F_{v}$ denote the total driving force at the wheels and

$$
P_{v}=v F_{v}
$$

the corresponding total wheel power. $F_{v}$ and $P_{v}$ are positive during traction (when the energy is transferred from the powertrain to the vehicle) and negative during braking. Therefore,

$$
P_{t}=P_{v}, \quad \forall P_{v} \geq 0,
$$

and because of the mechanical brakes, $P_{h}$ :

$$
P_{t}=P_{v}-P_{h}, \quad \forall P_{v}<0 .
$$

In this work, the mechanical brakes are simply modeled as power withdrawal, that is as a source of negative power $P_{h}<0$. Furthermore, as commonly assumed in energy management studies (see for example [24] and [33]), the regenerative braking is not restricted by the braking distribution between front and rear axles, such that all the braking power is recoverable and it is only restricted by the SS power charging limit, $P_{b, \min }$. As such, the mechanical braking power is derived as follows:

$$
P_{h}=\min \left(0, P_{v}-P_{b, \min } / \eta_{p l}\right)
$$

The vehicle longitudinal dynamics are described by:

$$
m_{v} \frac{d}{d t} v=\left(F_{v}-F_{R}-F_{D}\right)
$$

where $m_{v}$ is the vehicle mass, and $F_{R}=f_{R} m_{v} g$ and $F_{D}=$ $f_{D} v^{2}$ represent the resistance forces due to the tire rolling and aerodynamic drag, respectively. Finally, the traveled distance $s$ is calculated by integrating the longitudinal speed:

$$
\frac{d}{d t} s=v .
$$

The two sources (PS and SS) and the propulsion load branches are integrated at the DC-link with the corresponding power balance given as follows:

$$
P_{r}+P_{b}=P_{p l}=\eta_{p l}^{-\operatorname{sign}\left(P_{t}\right)} P_{t} .
$$

The main characteristic parameters of the vehicle model are summarized in Table I, where the parameters are chosen to represent a medium-size passenger car with non-plug-in HEV powertrain.

TABLE I

MAIN Vehicle MODEL PARAMETERS

\begin{tabular}{lcl}
\hline \hline symbol & value & description \\
\hline$m_{v}$ & $1500 \mathrm{~kg}$ & vehicle mass \\
$f_{R}$ & 0.01 & rolling resistance coefficient \\
$f_{D}$ & $0.47 \mathrm{~kg} / \mathrm{m}$ & aerodynamic drag coefficient \\
$\eta_{t}$ & 0.96 & efficiency of the transmission \\
$g_{t}$ & 10 & transmission ratio \\
$q_{f 0}$ & $0.12 \mathrm{~g} / \mathrm{s}$ & idling fuel mass rate \\
$\alpha_{f}$ & $0.059 \mathrm{~g} / \mathrm{kW} / \mathrm{s}$ & power transformation factor \\
$Q_{\max }$ & $5 \mathrm{Ah}$ & battery capacity \\
$R_{b}$ & $0.2056 \Omega$ & battery internal resistance \\
$V_{o c}$ & $300 \mathrm{~V}$ & battery open circuit voltage \\
$\eta_{r}, \eta_{i}, \eta_{d c}$ & 0.96 & efficiency of converters \\
$P_{b, \min } / \max$ & $-15 / 30 \mathrm{~kW}$ & SS power limits \\
$\mathrm{SOC}$ & $0.5 / 0.8$ & battery SOC limits \\
$P_{r, \max } / \max$ & $75 \mathrm{~kW}$ & PS power limit \\
\hline \hline
\end{tabular}




\section{B. Problem formulation and the benchmark solution}

This section characterizes the optimal control problem addressed in this paper, and reviews the possible solution methods, including a benchmark solution.

The main objective is to minimize the fuel consumption $m_{f}(T)$ for a driving mission by finding an appropriate driving speed and energy source power split, subject to various state and control constraints. The intuitive method to solve such problem is the joint optimization scheme (OCP-Joint) that formulates the whole problem as a single OCP, thus both aspects (vehicle speed and power-split between the engine and battery) can be solved simultaneously. By applying the conventional speed optimization approaches (for conventional or electric vehicles as reviewed in the Introduction), it is also possible to divide the concerned problem into two subproblems, 1) driving speed optimization (OCP-S), and 2) energy management optimization (OCP-EM), which are solved individually in successive steps and combined a posteriori, yielding a counterpart strategy (OCP-Split). In Fig. 4, both optimization mechanisms are illustrated.

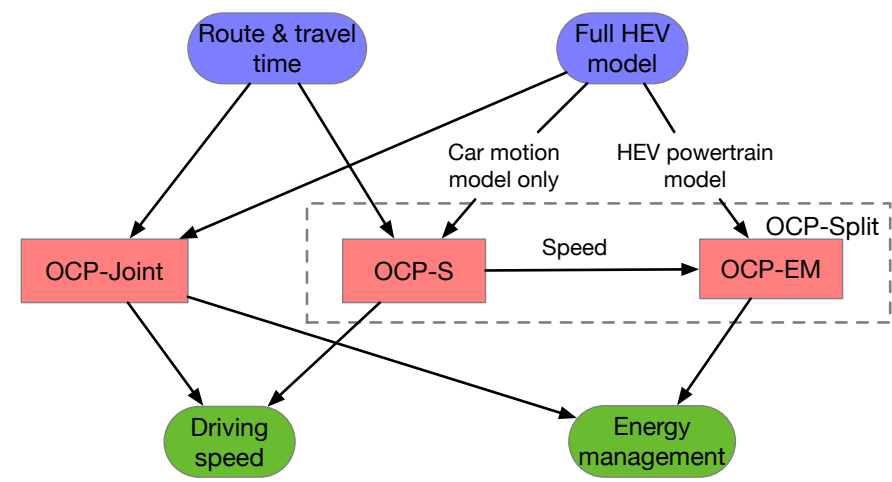

Fig. 4. Optimization problems formulated in this paper for a series HEV.

A major benefit in calculation time can be expected when solving the whole optimization problem in two steps as carried out in the OCP-Split, thus relieving the computational burden caused by the joint optimization of OCP-Joint. However, the main challenge for the OCP-Split is to decouple the EM and the vehicle speed optimization, while maintaining sufficient information of the powertrain and its EM in the OCP-S. If that is achieved, the speed profile found by the OCP-S will tend to the solution of OCP-Joint, and thus the overall optimality of the OCP-Split will be improved. In the next section, a novel OCP-Split algorithm aiming to realize this potential is proposed and benchmarked against the OCP-Joint by numerical simulation examples.

Prior to the introduction of the novel scheme, the benchmarking solution OCP-Joint is formulated next. The associated optimal control problem is:

$$
\begin{array}{cl}
\underset{\mathbf{u}}{\operatorname{minimize}} & J(\mathbf{x}, \mathbf{u}) \\
\text { subject to: } & \frac{d}{d t} \mathbf{x}=\mathbf{f}(\mathbf{x}, \mathbf{u}, t) \\
& \psi(\mathbf{x}, \mathbf{u}, t) \leq \mathbf{0} \\
& \mathbf{b}(\mathbf{x}(0), \mathbf{x}(T))=\mathbf{0}
\end{array}
$$

where $T$ denotes the total traveling time and $J(\mathbf{x}, \mathbf{u})=m_{f}(T)$ represents the main objective. The vector $\mathbf{x}$ represents the system state vector, which evolves according to the dynamic system (18b), and the vector $\mathbf{u}$ represents the vector of control inputs. The inequality constraints and the boundary conditions are taken into account by (18c) and (18d).

To ensure smooth vehicle motion and to avoid unrealistic jerky maneuvers, the vehicle is controlled in terms of the jerk decision variables $\mathbf{u}=\left[j_{r}, j_{b}, j_{v}\right]^{\top}$, which are the first derivative of the associated PS branch, SS branch and total driving forces, respectively $F_{r}, F_{b}$ and $F_{v}$. In this context, the source power values can be immediately obtained by $P_{r}=v F_{r}$ and $P_{b}=v F_{b}$. By combining the dynamic equations (1), (5), (15), and (16), and the dynamics of $F_{r}$, $F_{b}$ and $F_{v}$, the overall dynamic system is:

$$
\frac{d}{d t}\left(\begin{array}{c}
s \\
v \\
m_{f} \\
\mathrm{SOC} \\
F_{r} \\
F_{b} \\
F_{v}
\end{array}\right)=\left(\begin{array}{c}
v \\
\frac{F_{v}-F_{R}-F_{D}}{m_{v}} \\
\frac{q_{f 0}+\alpha_{f} v F_{r}}{-V_{o c}+\sqrt{V_{o c}^{2}-4 v F_{b} R_{b} / \eta_{d c}^{\operatorname{sign}\left(P_{b}\right)}}} \\
2 R_{b} Q_{\max } \\
m_{v} j_{r} \\
m_{v} j_{b} \\
m_{v} j_{v}
\end{array}\right) .
$$

with the state variable $\mathbf{x}=\left[s, v, m_{f} \text {, SOC, } F_{r}, F_{b}, F_{v}\right]^{\top}$.

The inequality constraints of OCP-Joint are as follows. First, the vehicle speed and acceleration are constrained for safety and comfort purposes. Specifically,

$$
0 \leq v \leq v_{\max }
$$

where $v_{\max }$ is the legal speed limit. Instead of the commonly used ellipse of adherence of tires, the longitudinal and lateral accelerations are constrained within an acceleration diamond, as everyday drivers use accelerations remarkably smaller than adherence limits [39]. The constraint is described as follows:

$$
\left|\frac{F_{v} / m_{v}}{a_{x, \max }}\right|+\left|\frac{v^{2} \Theta}{a_{y, \max }}\right| \leq 1,
$$

where $\Theta$ is the curvature of the road center, calculated from its Cartesian coordinates $(x, y)$ as a function of the travelled distance $s$ :

$$
\Theta(s)=\sqrt{\left(\frac{d^{2} x}{d s^{2}}\right)^{2}+\left(\frac{d^{2} y}{d s^{2}}\right)^{2}} .
$$

The two numerators $F_{v} / m_{v}$ and $v^{2} \Theta$ are respectively the longitudinal and the lateral acceleration applied by the driver, while $a_{x, \max }$ and $a_{y, \max }$ are their maximum allowed values. To keep the operating conditions of the powertrain components inside their admissible range, the following inequality 
constraints are also imposed,

$$
\begin{array}{r}
F_{r}+F_{b} \geq \eta_{p l}^{\operatorname{sign}\left(-F_{v}\right)} F_{v} \\
-j_{b, \text { max }} \leq j_{b} \leq j_{b, \text { max }} \\
-j_{r, \text { max }} \leq j_{r} \leq j_{r, \text { max }} \\
-j_{v, \text { max }} \leq j_{v} \leq j_{v, \text { max }} \\
\mathrm{SOC}_{\text {min }} \leq \mathrm{SOC} \leq \mathrm{SOC}_{\text {max }} \\
0 \leq v F_{r} \leq P_{r, \text { max }} \\
P_{b, \text { min }} \leq v F_{b} \leq P_{b, \text { max }}
\end{array}
$$

The inequality constraint (23a) is proposed to reflect the power balance at the DC-link in (17). It is formulated as an inequality, rather than a (less computationally efficient) equality constraint, to account for both $F_{v} \geq 0$ and $F_{v}<0$ cases, for which $P_{t}=v F_{v}$ and $P_{t}>v F_{v}$ respectively (see (12) and (13)), while also $v F_{r}+v F_{b}$ is minimized to reduce the overall fuel consumption. Inequality conditions (23b)-(23d) are imposed with $j_{b, \max }=j_{r, \max }=j_{v, \max }=1 \mathrm{~m} / \mathrm{s}^{3}$ for smooth operation [32]. In this work, we let $\mathrm{SOC}_{\text {min }}=0.5$ and $\mathrm{SOC}_{\max }=0.8$ to ensure the battery is closely charge sustained throughout the mission. Moreover, the power limits of the energy sources are given in Table I.

The following boundary conditions are imposed to complete the formulation:

$$
\begin{aligned}
& s(0)=0, s(T)=l, \\
& v(0)=v(T)=0, \\
& \operatorname{SOC}(0)=\operatorname{SOC}(T)=0.65, \\
& F_{b}(0)=F_{b}(T)=0, \\
& F_{r}(0)=F_{r}(T)=0, \\
& F_{v}(0)=F_{v}(T)=0, \\
& m_{f}(0)=0,
\end{aligned}
$$

where $l$ is the length of the path, SOC is initialized at the middle of the allowed SOC range, and the other states are initialized at 0 to emulate the start of a driving mission. To cancel the equivalent fuel caused by the non-zero differences between initial and terminal conditions of $v$, SOC, $F_{b}, F_{r}$ and $F_{v}$, terminal conditions as presented in (24b)-(24f) are also employed.

\section{THE TWO-STEP OPTIMAL CONTROL STRATEGY}

A computationally efficient OCP-Split algorithm is proposed in this section by combining a novel OCP-S (named OCP-S-2) and a standard OCP-EM, where OCP-S-2 is the core contribution of this paper and the OCP-EM is formulated by using the speed profiles obtained by the speed optimization as the reference input. The overall two-step scheme is benchmarked against the OCP-Joint introduced in Section II-B and another OCP-Split benchmark that is formed by the driving speed optimization strategy (named OCP-S-1) proposed in [36] by the present authors and the same OCP-EM. OCP-S-1 is described next in this section followed by the introduction of the novel scheme.

\section{A. Driving speed optimization $(O C P-S)$}

The problem of finding the energy efficient driving speed of a conventional vehicle has already been studied in the literature. Nonetheless, the optimal solutions may not be compatible when it comes to an HEV.

1) OCP-S-1: Prior work of the present authors in [36] has proposed an enhanced OCP-S that formulates the possible energy recovery phenomenon of an HEV by a specialized objective function for the following state space model (a part of the full model (19) presented in the OCP-Joint):

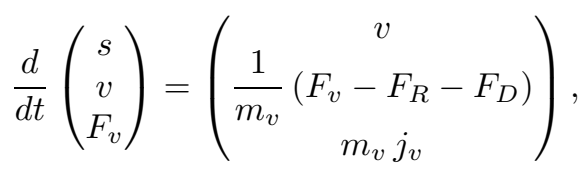

with three states $\mathbf{x}=\left[s, v, F_{v}\right]^{\top}$ and one input $\mathbf{u}=j_{v}$. The core idea has been to define an energy recovery factor $\rho \in$ $[0,1]$ as the ratio between regenerative braking power and the total braking power, such that the energy dissipation function becomes:

$$
J_{1}=\int_{0}^{T}\left\{\begin{array}{ll}
F_{v} & F_{v} \geq 0 \\
\rho F_{v} & F_{v}<0
\end{array} v d t=\int_{0}^{T} \max \left(\rho F_{v}, F_{v}\right) v d t .\right.
$$

Similarly to the OCP-Joint formulated in the previous section, the possible maneuvers are constrained by (20), (21), (23d), and

$$
P_{v} \leq P_{v, \max }=\left(P_{b, \max }+P_{r, \max }\right) \eta_{p l}
$$

where the total input power is bounded by a variable limit, depending on the individual power limits of the PS and the SS branches, and the instantaneous PL branch efficiency $\eta_{p l}$. Finally, the problem is completed by the boundary conditions (24a), (24b) and (24f).

As it can be noticed, the case $\rho=0$ corresponds to a conventional powertrain, while $\rho=1$ indicates that all braking energy is recovered as in a highly hybridized or fully electric vehicle. However, the speed profile solved by such an OCP$\mathrm{S}$ still cannot capture the essential behavior of the OCPJoint solution as the energy source power split and powertrain efficiencies are not incorporated. Consequently, the resulting OCP-Split in [36] falls behind OCP-Joint by over 5\% in terms of fuel economy.

2) $O C P-S-2$ : The new driving speed optimization strategy is introduced in this section. The core idea is to design an objective function that integrates the essential information and characteristics of regenerative braking and powertrain energy losses, without including the explicit powertrain model in (25). To this end, the following three steps are followed: 1) an analysis that finds the nature of the optimal energy source power split solution, 2) evaluation of the powertrain minimum power losses, informed by the nature of the optimal power split solution found in the first step, and 3) introduction of a new objective function that integrates the fuel energy and energy recovery with the powertrain losses characterized in the second step. 
Step 1: Given the vehicle driving speed (and acceleration), it is clear that the total driving force $F_{v}$, and consequently the associated power $P_{v}$, is uniquely determined:

$$
P_{v}=v\left(m_{v} a+F_{R}+F_{D}\right) \text {. }
$$

To proceed with the analysis, let us first introduce some useful notations and definitions. Consider two sets of time intervals respectively for the propulsive phase $\Phi \triangleq\left\{t \mid P_{v}(t) \geq 0\right\}$ and the energy recovery phase $\Psi \triangleq\left\{t \mid P_{v}(t)<0\right\}$, such that $\Phi \cup \Psi$ is the full time horizon $\{t \mid 0 \leq t \leq T\}$. Analogously, $\Phi$ can be divided into two subsets as $\Phi=\Phi_{d} \cup \Phi_{c}$, where $\Phi_{d} \triangleq$ $\left\{t \mid P_{v}(t) \geq 0, P_{b}(t) \geq 0\right\}$ and $\Phi_{c} \triangleq\left\{t \mid P_{v}(t) \geq 0, P_{b}(t)<0\right\}$. These subsets respectively collect the battery discharging and charging (by the ICE) intervals for all $t \in \Phi$.

By resorting to (12) and (17), the sum of PS and SS power is determined when $P_{v} \geq 0$ :

$$
P_{r}+P_{b}=\frac{P_{v}}{\eta_{p l}}, \quad \forall P_{v} \geq 0 .
$$

On the other hand, when $P_{v}<0$, by using (13) and (14), the PS and SS power is shown to be:

$$
P_{b}=\max \left(P_{v} \eta_{p l}, P_{b, \min }\right), P_{r}=0, \forall P_{v}<0 .
$$

The overall fuel consumption at the end of the mission can be obtained by integrating both sides of the fuel consumption model (1) over $[0, T]$, as follows:

$$
m_{f}(T)=q_{f 0} T+\alpha_{f} \int_{\Phi} P_{r} d t .
$$

Note that the integration of $P_{r}$ is done only for the $\Phi$ time interval, since for the $\Psi$ time interval $\left(P_{v}<0\right)$ the PS power $P_{r}=0$ (see (30)) and therefore this case does not contribute to the fuel consumption. By applying (3) and (29) in (31), we obtain:

$$
m_{f}(T)=m_{f 0}-\alpha_{f}\left(\int_{\Phi_{d}} \eta_{d c} P_{b} d t+\int_{\Phi_{c}} \frac{1}{\eta_{d c}} P_{b} d t\right),
$$

where

$$
m_{f 0}=q_{f 0} T+\alpha_{f} \int_{\Phi} \frac{P_{v}}{\eta_{p l}} d t
$$

is a fixed term, independent of the EM control. It is clear that one of the necessary conditions to minimize $m_{f}(T)$ in (32) is:

$$
\int_{\Phi_{c}} P_{b} d t=0
$$

since $\int_{\Phi_{c}} P_{b} d t \leq 0$. The condition (34) further implies that $m_{f}(T)$ is minimized if $\Phi_{c}=\emptyset$, indicating that for an optimal EM the battery is never charged directly by the PS, instead, the battery is only replenished by regenerative braking. In the literature correction schemes have been employed when calculating the SS efficiency, for example by post-multiplication of the SS discharging efficiency with the PS average efficiency, such that future power losses of the PS for replenishing the consumed SOC in the SS are accounted for [40]. However, the finding in (34) allows for our purposes to evaluate the efficiency/power loss of the SS independently of the PS efficiency, without applying any correction. The power losses of PS and SS are therefore computed by:

$$
\begin{aligned}
& P_{r, \text { loss }}=P_{f}-P_{r}=q_{L H V}\left(q_{f 0}+\alpha_{f} P_{r}\right)-P_{r}, \\
& P_{b, \text { loss }}=V_{o c} i_{b}-P_{b},
\end{aligned}
$$

where $q_{L H V}=42.6 \mathrm{MJ} / \mathrm{kg}$ is the diesel lower heating value.

Step 2: With reference to the preceding analysis of the optimal EM solution, the minimum power loss of the powertrain, including mechanical brakes, at each $P_{v}$ is now estimated to inform the formulation of the new OCP-S-2. Since the PS is a much more lossy component than the SS because of the ICE, the powertrain minimum losses are due to SS use only, where it is possible to employ the SS. The estimation of the power losses can therefore be considered in four $P_{v}$ ranges, with the associated power splits as follows:

$$
\begin{array}{lr}
P_{r}=P_{v} / \eta_{p l}-P_{b, \max }, P_{b}=P_{b, \text { max }}, & P_{v} / \eta_{p l}>P_{b, \max }, \\
P_{r}=0, P_{b}=P_{v} / \eta_{p l}, & 0 \leq P_{v} / \eta_{p l} \leq P_{b, \max }, \\
P_{r}=0, P_{b}=P_{v} \eta_{p l}, & P_{b, \text { min }} \leq P_{v} \eta_{p l}<0, \\
P_{r}=0, P_{b}=P_{b, \text { min }}, & P_{v} \eta_{p l}<P_{b, \min .} .
\end{array}
$$

As it can be noticed, during the propulsive phase $\Phi$, the minimum losses are when the powertrain is operated in pure electric mode until $P_{v} / \eta_{p l}>P_{b, \max }$, and if $P_{v}$ further increases, the PS is required to be activated to meet the demand, and therefore its losses will apply in addition to the SS losses. Conversely, the power flow of the PS, the SS and the mechanical brakes simply follows (14) and (30) when $P_{v}<0$. Thus, by making use of (35) and the fact $\eta_{p l}<1$, the lower bound of the power loss in each power range is as follows (obtained for $\eta_{p l}=1$ ):

$$
P_{l o s s, 1}=\left\{\begin{array}{lr}
q_{L H V}\left(q_{f 0}+\alpha_{f} P_{r}\right)-P_{r} & \\
\quad+V_{o c} i_{b, \max }-P_{b, \max }, & P_{v}>P_{b, \max }, \\
V_{o c} i_{b}-P_{b}, & 0 \leq P_{v} \leq P_{b, \max }, \\
V_{o c} i_{b}-P_{b}, & P_{b, \min } \leq P_{v}<0, \\
V_{o c} i_{b, \min }-P_{v}, & P_{v}<P_{b, \min },
\end{array}\right.
$$

where $i_{b, \max }$ and $i_{b, \min }$ are the battery currents respectively for $P_{b, \max }$ and $P_{b, \min }$, which can be calculated from (4). By substituting $P_{r}, P_{b}$ from (36) and $i_{b}$ from (4), this power loss can be expressed as a piecewise function of $P_{v}$ only, as illustrated in Fig. 5.

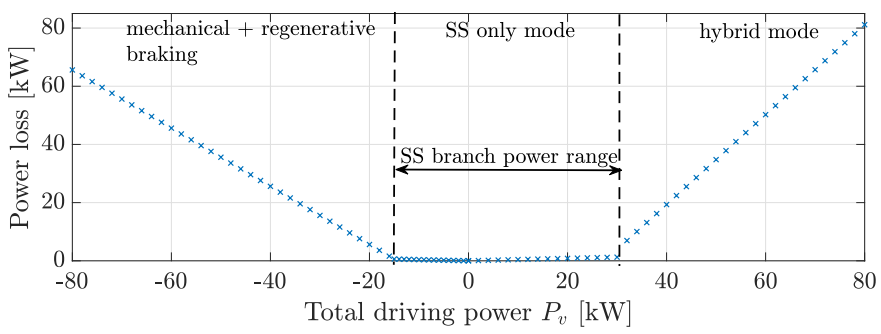

Fig. 5. Analytic powertrain minimum power losses including mechanical braking loss for varied total driving power, $P_{v}$. The PL branch efficiency, $\eta_{p l}$, is assumed to be 1 . The associated power split is as follows: when $P_{v} \geq 0$, only the SS is used until $P_{b, \max }$ is reached, and for higher $P_{v}$ values the powertrain is operated in PS and SS hybrid mode with $P_{v}=P_{b, \max }+P_{r}$; when $P_{v}<0$ regenerative braking takes place, and mechanical braking power is not applied until the total braking power becomes smaller than $P_{b, \text { min }}$. 
It is clear that the actual minimum power loss diverges from the analytic minimum profile shown in Fig. 5 as the PL branch efficiency $\eta_{p l}$ decreases from 1 . To avoid the inaccuracy at a low $\eta_{p l}$ (for example, when the vehicle is accelerating hard at low speed), the power loss of the PL branch is also evaluated, resorting to the commonly used closed-form expression [1] for estimating the electric power supplied to or generated by the motor:

$$
P_{i}=c_{1} \tau_{l m} \omega_{m}+c_{2} \tau_{l m}^{2} .
$$

In view of (38), the fixed transmission ratio, and the constant efficiencies of the transmission and the inverter, the PL branch power can be linked to $P_{v}$ by:

$$
P_{p l}=d_{1} P_{v}+d_{2} \tau_{v}^{2}
$$

where $\tau_{v}=P_{v}^{2} /\left(\mathrm{g}_{t} v\right)^{2}=F_{v}^{2} / \mathrm{g}_{t}^{2}$ is the load torque at the wheels, and the parameters $d_{1}$ and $d_{2}$ are obtained by fitting the model (39) to $P_{p l}\left(P_{v}, v\right)$ respectively for motoring $\left(P_{p l}\left(P_{v}, v\right)=P_{v} / \eta_{p l}\left(P_{v}, v\right)\right)$ and generating $\left(P_{p l}\left(P_{v}, v\right)=\right.$ $\left.P_{v} \eta_{p l}\left(P_{v}, v\right)\right)$ phases. As such the power loss of the PL branch is estimated by terms of the form:

$$
P_{l o s s, 2}=P_{p l}-P_{v}=\left(d_{1}-1\right) P_{v}+d_{2} \tau_{v}^{2} .
$$

In order to include the relationship between $P_{v}$ and power losses into the speed optimization without a significant increase in computational effort, let us rewrite the total driving power $P_{v}$ as the combination of four individual segments, such that:

$$
P_{v}=\sum_{i=1}^{4} P_{v, i}
$$

with

$$
\begin{array}{ll}
P_{v, 1} \leq 0, & P_{b, \min } \leq P_{v, 2} \leq 0, \\
0 \leq P_{v, 3} \leq P_{b, \max }, & 0 \leq P_{v, 4} \leq P_{r, \max } .
\end{array}
$$

As such the piecewise nonlinear function (37) is fitted by four piecewise linear functions of $P_{v, i}, i=1, \cdots, 4$, respectively, expressed as follows:

$$
P_{l o s s, 1}=\sum_{i=1}^{4} a_{i} P_{v, i}+b_{i}
$$

where $a_{2}=a_{3}=0.04, a_{1}=1, a_{4}=1.545$ are constants obtained using the least square regression method. Moreover, by defining $F_{v, i}=P_{v, i} / v$ the power associated force, the PL power loss (40) is:

$$
\begin{aligned}
P_{l o s s, 2}= & \left(d_{1}^{\prime}-1\right)\left(P_{v, 1}+P_{v, 2}\right)+d_{2}^{\prime}\left(F_{v, 1}+F_{v, 2}\right)^{2} / \mathrm{g}_{t}^{2} \\
& +\left(d_{1}-1\right)\left(P_{v, 3}+P_{v, 4}\right)+d_{2}\left(F_{v, 3}+F_{v, 4}\right)^{2} / \mathrm{g}_{t}^{2},
\end{aligned}
$$

where the losses for both the motoring and generating phases are separately accounted for. With reference to the PL branch efficiency map (obtained by multiplying the motor efficiency map in Fig. 2 with $\eta_{t}$ and $\eta_{i}$ ), it is immediate to obtain:

$$
\begin{array}{ll}
d_{1}=1.049, d_{2}=0.033, & \text { if } P_{v} \geq 0, \\
d_{1}^{\prime}=0.954, d_{2}^{\prime}=0.03, & \text { if } P_{v}<0,
\end{array}
$$

by polynomial regression fit.
Step 3: The determination of the power loss relationships presented, enables to design a new objective function for the OCP-S-2 that includes: a) the fuel energy $\left.\int_{0}^{T} \max \left(P_{v}, 0\right) d t, \mathrm{~b}\right)$ the energy recovery $\int_{0}^{T} \min \left(P_{v}, 0\right) d t$, and c) the terms that penalize the energy loss due to the power withdrawal from both energy sources. The objective function is:

$$
\begin{aligned}
J_{2}= & \int_{0}^{T}\left(P_{v}+P_{l o s s, 1}+P_{l o s s, 2}\right) d t \\
= & \int_{0}^{T} \sum_{i=1}^{4} a_{i} P_{v, i}+d_{1}^{\prime}\left(P_{v, 1}+P_{v, 2}\right)+d_{1}\left(P_{v, 3}+P_{v, 4}\right) \\
& +d_{2}^{\prime}\left(F_{v, 1}+F_{v, 2}\right)^{2} / \mathrm{g}_{t}^{2}+d_{2}\left(F_{v, 3}+F_{v, 4}\right)^{2} / \mathrm{g}_{t}^{2} d t \\
& \frac{d}{d t}\left(\begin{array}{c}
s \\
v \\
F_{v, 1} \\
F_{v, 2} \\
F_{v, 3} \\
F_{v, 4}
\end{array}\right)=\left(\begin{array}{c}
1 \\
m_{v}
\end{array}\left(\begin{array}{c}
\left.\sum_{i=1}^{4} F_{v, i}-F_{R}-F_{D}\right) \\
m_{v} j_{v, 1} \\
m_{v} j_{v, 2} \\
m_{v} j_{v, 3} \\
m_{v} j_{v, 4}
\end{array}\right)\right.
\end{aligned}
$$

with augmented state and input vectors: $\mathbf{x}=$ $\left[s, v, F_{v, 1}, F_{v, 2}, F_{v, 3}, F_{v, 4}\right]^{\top}$ and $\mathbf{u}=\left[j_{v, 1}, j_{v, 2}, j_{v, 3}, j_{v, 4}\right]^{\top}$.

Analogously to the OCP-S-1 formulated in the previous section, OCP-S-2 is subject to the constraints (20), (21), (41), $j_{v, i} \leq\left|j_{v, \max }\right|, i=1, \cdots, 4$, and,

$$
\sum_{i=1}^{4} P_{v, i} \leq P_{v, \max }=\left(P_{b, \max }+P_{r, \max }\right) \eta_{p l}
$$

which is inferred from (27). The boundary conditions (24a), (24b) and $F_{v, i}(0)=F_{v, i}(T)=0, i=1, \ldots, 4$ are also imposed to complete the formulation.

\section{B. Energy management optimization (OCP-EM)}

The problem of EM optimization for an HEV while following a predefined speed profile is standard with a rich literature (see, for example [14], [16] and the references cited therein). The problem has also been specifically formulated in numerous prior studies as an OCP in the form of (18) (see, for example [41]).

In this work, the OCP-EM is formulated with reference to the OCP-Joint presented in Section II-B. For a given speed profile (which can be obtained by the OCP-S), the OCP-EM is formulated to find the powers $P_{r}$ and $P_{b}$ provided by the PS and SS respectively, which minimize fuel consumption. Therefore the cost to be minimized is:

$$
J=m_{f}(T),
$$

and the state space model is:

$$
\frac{d}{d t}\left(\begin{array}{c}
m_{f} \\
\mathrm{SOC} \\
F_{r} \\
F_{b}
\end{array}\right)=\left(\begin{array}{c}
-q_{f 0}+\alpha_{f} v F_{r} \\
\frac{V_{o c}^{2}-4 v F_{b} R_{b} / \eta_{d c}^{\operatorname{sign}\left(P_{b}\right)}}{2 R_{b} Q_{\max }} \\
m_{v} j_{r} \\
m_{v} j_{b}
\end{array}\right)
$$


where $\mathbf{x}=\left[m_{f}, \mathrm{SOC}, F_{r}, F_{b}\right]^{\top}$ and $\mathbf{u}=\left[j_{r}, j_{b}\right]^{\top}$ are formed by a part of the elements of the full model used for OCPJoint. To complete the problem, the inequality constraints (23a)-(23c), (23e)-(23g) and the boundary conditions (24c), (24d), (24e), (24g) are also imposed. Note that the right hand side of (23a), $\eta_{p l}\left(F_{v}, v\right)^{\operatorname{sign}\left(-F_{v}\right)} F_{v}$, is a given function of time determined by the chosen speed profile $v(t)$, with $F_{v}(t)$ obtained from (15).

All the OCPs formulated in this paper are addressed by the MATLAB-based software package GPOPS-II, which is a tool for solving general-purpose optimal control problems [42]. GPOPS-II contains the machinery for transcribing a trajectory optimization problem into an NLP and incorporates the well developed solver, IPOPT, to solve the resulting NLP. To set up an optimization problem in GPOPS-II, the initial segmentations and number of collocation points within each segment need to be initialized, and GPOPS-II will automatically refine the grid mesh during the computation process.

\section{Simulation RESUltS AND DISCUSSION}

Two case studies are presented in this section to evaluate the performance of the proposed two-step optimal control strategy, formed by OCP-S-2 and OCP-EM (OCP-Split-2), in the context of "free driving" scenarios. For benchmarking purposes, the proposed algorithm is compared with the OCPJoint formulated in Section II-B and with the existing OCPSplit algorithm [36] formed by OCP-S-1 and OCP-EM (OCPSplit-1).

\section{A. Case Study I}

In this example the vehicle is requested to drive only for $1 \mathrm{~km}$ on a straight and flat road with prescribed traveling time $T=60$ s (i.e., average speed $60 \mathrm{~km} / \mathrm{h}$ ). The speed limit is assumed to be much higher than the average speed in the first instance, such that it does not affect the solution, to expose the nature of the 'untruncated' optimal driving speed in each OCP case. Moreover, such a problem setup can highlight the important characteristics of each optimal control solution, while the results also provide practical insight since an arbitrary path can be reasonably treated as the combination of multiple straight segments [32].

In Fig. 6, the optimal speed profile solved by the proposed OCP-S-2 is illustrated and compared with various solutions of the OCP-S-1 and the speed profile from the OCP-Joint. As it can be noticed, when $\rho=0$, the OCP-S- 1 emulates the driving speed optimization of a conventional vehicle. The optimal profile starts from a rapid acceleration to a maximum velocity, after which the vehicle mildly decelerates by coasting for a considerable time to a relatively low speed, at which point intensive braking is applied to stop the vehicle. Such driving behavior coincides with the PnG strategy found in the literature for conventional vehicles. By increasing the energy recovery factor $\rho$, the most fuel-efficient driving speed profile changes and eventually when $\rho=1$ it shifts to a more regular profile that suggests the driver to accelerate to a cruise speed value, then to stay at this speed until the destination approaches, as drivers normally do. Although the extra degree

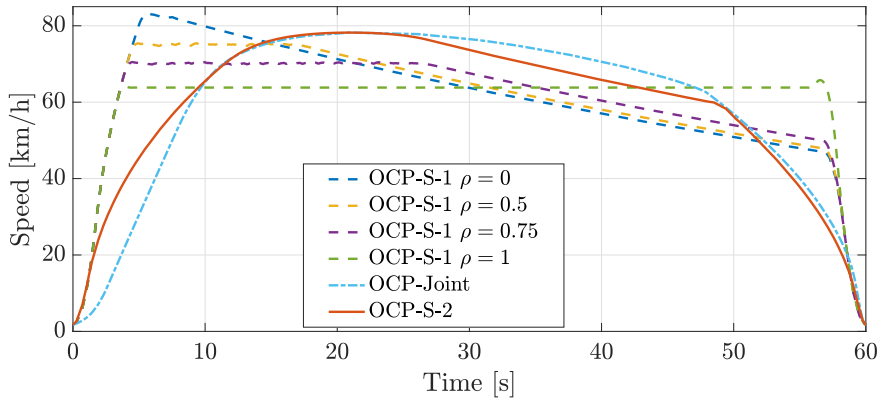

Fig. 6. Optimal speed profiles solved by OCP-Joint, the OCP-S-1 [36] with different $\rho$ and the proposed OCP-S-2 for a $1 \mathrm{~km}$ straight road.

of freedom offered by $\rho$ allows the OCP-S- 1 to find various solutions depending on the energy recovery capability, in all $\rho$ cases noticeable differences can be observed as compared to the joint optimization solution. This can be understood that the most-fuel efficient driving speed is highly influenced by the characteristics of the powertrain, such as the energy source efficiency and power-split, which are ignored by OCPS-1 but are taken into account by OCP-Joint. In particular, it is not efficient to apply hard acceleration or deceleration at the beginning or end of a mission, as in that case the energy source, especially the ICE is inefficiently operated or the battery reaches its charging limit and energy is wasted in mechanical braking.

Nevertheless, it is expected that the speed profile obtained by OCP-S-1 with $\rho=1$ is close-to-optimal in the context of a full electric vehicle as the efficiency of the associated electric branch (battery and electric motor) is much higher than that of the ICE, unless the battery is inadequately sized and mechanical braking is required to be used. Therefore, it can be said that without energy recovery eco-driving requires a pulse and glide type profile, which is not natural and difficult to be implemented by drivers because the proper identification of the maximum speed and braking points requires a lot of anticipation. In contrast, energy recovery does not only reduce fuel consumption, but also allows the driver to drive more regularly without behavioral adaptation.

On the other hand, the speed profile solved by the proposed OCP-S-2 is able to capture the essential features of the OCPJoint benchmark solution, and therefore it is much closer to it as compared to the OCP-S-1. The comparison emphasizes the importance of including the various losses of the HEV powertrain in the OCP-S objective function as formulated in (43). Further insight into the effectiveness of the new OCP-S2 scheme can be obtained by comparing the resulting power split solution of OCP-Split-2, with that of the OCP-Split-1 for $\rho=0.5$ (that leads to a similar peak speed to OCP-S-2) and the OCP-Joint, as shown in Fig 7.

It is illustrated that following the profiles obtained by OCP-S-1 requests from both energy sources very high power withdrawal with short duration at the start of the mission, while the other two OCP methods use much less power (as milder acceleration is applied, as seen in the corresponding speed profiles), which is beneficial to the powertrain efficiency according to the analysis performed in the previous section 

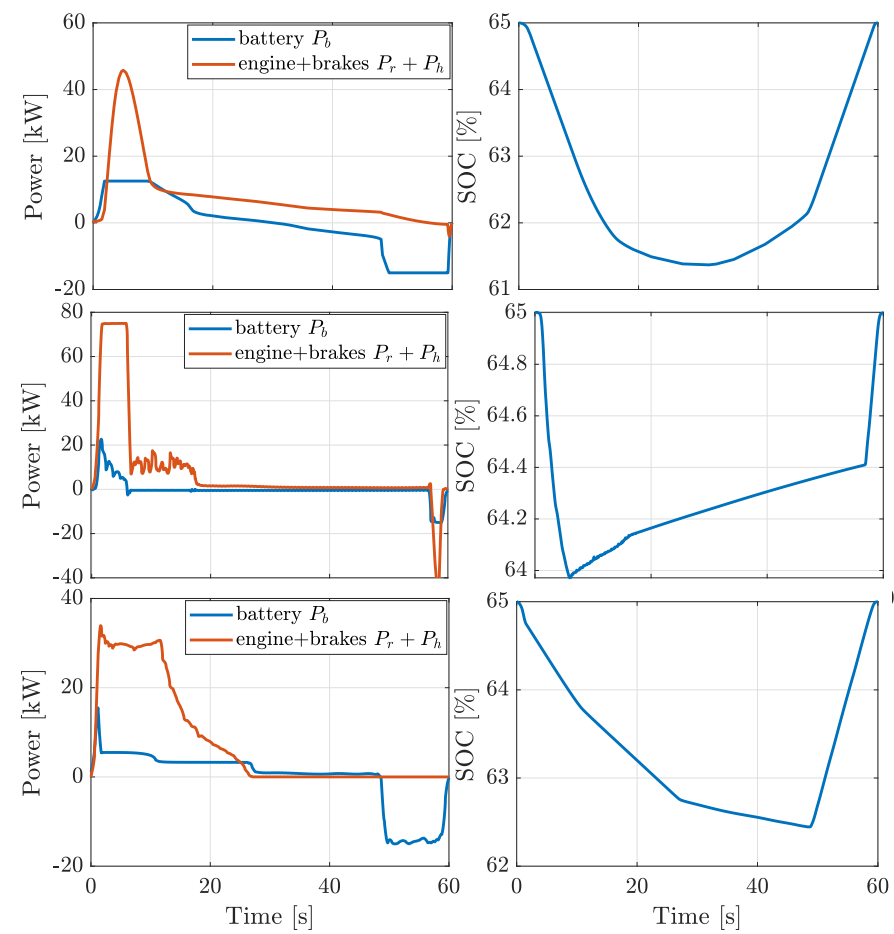

Fig. 7. Power flow and battery SOC profiles solved by the OCP-Joint (top), the OCP-Split-1 [36] for $\rho=0.5$ (middle) and the proposed OCP-Split-2 (bottom) for a $1 \mathrm{~km}$ straight road.

(see Fig. 5). A further observation is made by referring to the higher speed of the OCP-S-2 as compared to that of OCPJoint in the first $10 \mathrm{~s}$ in Fig. 6, and the apparent opposite to the expected trend in the power magnitudes of the two OCPs in the same time period in Fig. 7. This is explained by considering that even though the peak power values of OCP-Split-2 are lower, these are reached at an earlier time when OCP-Joint still has low power values, which lead to a higher initial acceleration for OCP-S-2, but which subsequently becomes less than that of OCP-Joint as the OCP-Joint power values rise to their higher peak values at a slightly later time. This apparent difference in the power profile behavior of the two OCPs is related to the (necessary) smoothing functions used for $\operatorname{sign}(\cdot)$ in the OCP-Joint numerical formulation in order to manage its computational complexity and convergence to a solution, which consequently lead to smoother power profiles. Such smoothed solutions may affect the optimality of the joint optimization solution, as it will be discussed later in this Section. By further observing the behavior of the power profiles towards the end of the mission, it is clear that as compared to the OCP-Split-1, braking is applied much earlier and for longer overall duration in OCP-Joint and OCP-Split2 cases. Therefore, lower magnitudes of braking power are adequate to stop the vehicle in OCP-Joint and OCP-Split2 , removing the need to use substantial mechanical braking power (as can be seen in the OCP-Split-1 solution), which is totally wasted.

In terms of the SOC profiles shown in Fig. 7, all the profiles are strictly CS at the end of the mission, as expected from the defined boundary conditions. The solution of OCP-Split1 has the lowest depth of discharge which is explained as follows: a) for a given speed profile, as in OCP-EM and consequently OCP-Split- 1 , the battery is charged only from regenerative braking, as derived in Section III-A2, b) OCPSplit-1 solutions tend to apply large braking power for a short duration, as can be seen in Fig. 7, and therefore regenerative braking tends to get truncated because of the battery charging power limit, c) hence only small amounts of regenerative braking energy can be collected, implying that only small amounts of battery energy can be discharged to remain CS. Therefore, the overall usage of the battery and consequently the depth of discharge is very limited. In contrast, OCP-Split2 and OCP-Joint make more use of the battery by avoiding large magnitudes of braking power, due to the knowledge they have of the power losses associated with such braking power magnitudes. Moreover, between them, the OCP-Split-2 solution has a slightly lower depth of discharge throughout the mission as compared to the OCP-Joint, which is more compatible with the aim of CS operation.

Finally, the fuel consumption of each method is evaluated as well as the average computation time required by each strategy, and shown in Table II. By using OCP-Split-1 as a

TABLE II

FUEL CONSUMPTION RESULTS WITH COMPARISONS OF THE TWO OCP-SPLIT WITH OCP-JOINT AND AVERAGE COMPUTATION TIME OF EACH METHOD FOR A 1 KM STRAIGHT ROAD. THE CPU USED FOR COMPUTATION IS INTEL XEON E5-1650, 3.5 GHz.

\begin{tabular}{lccc}
\hline \hline & $\begin{array}{c}\text { fuel economy } \\
{[\mathrm{L} / 100 \mathrm{~km}]}\end{array}$ & fuel increase & $\begin{array}{c}\text { running time } \\
{[\mathrm{s}]}\end{array}$ \\
\hline OCP-Joint [32] & 4.36 & 0 & 62.12 \\
OCP-Split-1 [36] & 4.71 & $7.87 \%$ & 5.71 \\
OCP-Split-2 & 4.28 & $-1.8 \%$ & 11.34 \\
\hline \hline
\end{tabular}

benchmark, vehicle fuel economy greatly benefits from the joint optimization. As shown, the OCP-Joint saves $7.87 \%$ of fuel in comparison to the OCP-Split-1, however, the required computation time of OCP-Joint is about 10 times longer. The OCP-Split-2 has significantly improved optimality as compared to OCP-Split-1, with an impressive $9.01 \%$ fuel saving for the $1 \mathrm{~km}$ straight mission, with only marginally higher computation time. It is worth noting that the final fuel usage of the OCP-Split-2 is even less than the result of OCP-Joint, with about $1.8 \%$ improvement (and about 6 times less computation time). This is attributed to the precision of implementation. As mentioned previously, non-smooth $\operatorname{sign}(\cdot)$ functions used in the representation of the system dynamics (with bidirectional power flow) are smoothed by approximations, which affect the accuracy of the optimal solution. In this context, the split optimization strategy is less influenced by such approximations, since the PL branch that contains the bi-directional power flow can be absorbed exactly in the PL power input to the OCP-EM part of the split optimization scheme, and hence it can be isolated from the optimization scheme. More specifically, given a speed profile, the right hand side of (23a), $\eta_{p l}\left(F_{v}, v\right)^{\operatorname{sign}\left(-F_{v}\right)} F_{v}$ can be uniquely computed by $v(t)$ and $F_{v}(t)$ (obtained from (15)). 


\section{B. Case Study II}

A more realistic example is considered in this subsection to further evaluate the effectiveness and robustness of the proposed two-step algorithm. The vehicle mission corresponds to a rural route of $7.2 \mathrm{~km}$, as shown in Fig. 8. The road is

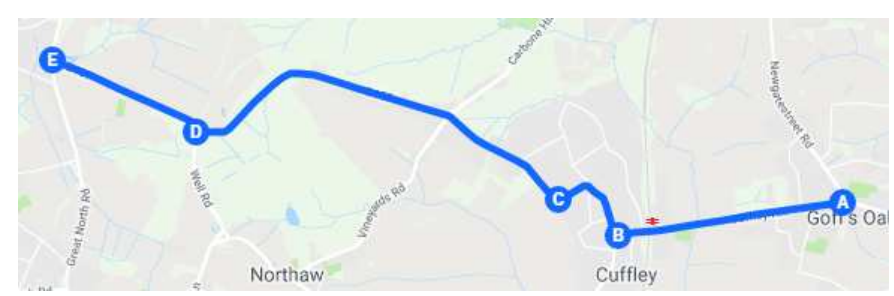

Fig. 8. $7.2 \mathrm{~km}$ route selected for the vehicle mission. http://tiny.cc/geve $7 \mathrm{y}$.

assumed to be flat and its geometry, defined by the longitude and latitude coordinates, is exported from Google Maps and converted into the curvature model (22). A variable upper speed limit is imposed throughout the journey according to the real conditions (legal speed limit) of the chosen path. Road traffic is not considered, therefore the speed is not constrained to the behavior of other vehicles. The trip is requested to be accomplished in $480 \mathrm{~s}$, which corresponds to an average speed of the vehicle of $54 \mathrm{~km} / \mathrm{h}$.

The optimal speed profiles solved by the three optimization schemes already compared in Section IV-A are shown in Fig. 9. As it can be seen, all the solutions tend to follow the two lower speed limits at $48.28 \mathrm{~km} / \mathrm{h}$ and $64.37 \mathrm{~km} / \mathrm{h}$, but not the highest one at $80.50 \mathrm{~km} / \mathrm{h}$. This can be understood that velocity optimization naturally minimizes the speed variance throughout the mission to avoid aggressive or unnecessary acceleration and deceleration, which usually lead to less efficient vehicle and powertrain operation. Moreover, the optimal solution from each optimization scheme is essentially compatible with its associated profile pattern illustrated in the $1 \mathrm{~km}$ case. For example, the solution of OCP-S-1 applies a much higher acceleration than the other two schemes at the beginning of the mission and after passing a corner (for example B, C, and D) or a rising edge of the speed limit (for example the one that appears at $2.8 \mathrm{~km}$ ). At the same time, between two successive intersections or speed limit changes, the profile is very close to that of the $1 \mathrm{~km}$ case, as it can be noticed from B to C, from $\mathrm{D}$ to $\mathrm{E}$ and from the speed limit change at $2.8 \mathrm{~km}$ to $\mathrm{D}$. It is also clear that as compared to the OCP-S-1, the speed solution of the OCP-S-2 is less aggressive and closer to the joint optimization solution, further verifying the findings with the $1 \mathrm{~km}$ case.

The effectiveness of the OSP-S-2 is further clarified by comparing in Fig. 10 the power flow and SOC profiles of the OCP-Joint and the two OCP-Split algorithms that are driven by OCP-S-1 and OCP-S-2, respectively. Similarly to the $1 \mathrm{~km}$ case, OCP-Split-1 requests higher propulsive power and applies noticeable mechanical braking during the mission. The OCP-Split-2 captures the main features of the OCPJoint power split solution, which operates the energy sources more efficiently at a medium level of propulsive power and with no noticeable mechanical braking (all braking power is regenerated), as a consequence of the driving profile obtained by OCP-S-2 (see Fig. 9). The similarity between the OCPSplit-2 and the OCP-Joint can also be identified from their SOC profiles, which have analogous tendencies.

Finally, the results of fuel usage and computation time of each method are shown in Table III. The OCP-Split-1 consumes $11.3 \%$ more fuel as compared to the OCP-Joint, while the OCP-Split-2 achieves a significant improvement over OCP-Split-1 and lags the OCP-Joint by only $4.95 \%$. However, the required computation time can be quartered by using the OCP-Split-2 rather than OCP-Joint. By comparing the outcomes presented in both case studies I and II, it is expected to gain more fuel benefit from OCP-Joint over OCP-Split as the complexity of the problem increases by involving more practical aspects such as road gradient and traffic. However, at the same time it is expected that the OCPJoint will run into infeasibility due to the increased overall complexity much earlier than OCP-Split. Hence OCP-Slit-2 offers a computationally and fuel efficient solution to problems that OCP-Joint will not be able to tackle.

TABLE III

FUEL CONSUMPTION RESULTS WITH COMPARISONS OF THE TWO OCP-SPLIT WITH OCP-JOINT AND AVERAGE COMPUTATION TIME OF EACH METHOD FOR THE 7.2 KM RURAL ROUTE.

\begin{tabular}{lccc}
\hline \hline & $\begin{array}{c}\text { fuel economy } \\
{[\mathrm{L} / 100 \mathrm{~km}]}\end{array}$ & fuel increase & $\begin{array}{c}\text { running time } \\
{[\mathrm{s}]}\end{array}$ \\
\hline OCP-Joint [32] & 3.22 & 0 & 1344 \\
OCP-Split-1 [36] & 3.59 & $11.3 \%$ & 238 \\
OCP-Split-2 & 3.38 & $4.95 \%$ & 347 \\
\hline \hline
\end{tabular}

\section{CONCLUSION}

A fundamental analysis of series hybrid electric vehicle (HEV) powertrain losses and optimal energy management (EM) solutions leads to the introduction of a novel driving speed optimization strategy for series HEVs. The strategy is formulated as a "free driving" optimal control problem with the mission defined in terms of traveling time and route characteristics, which are easily available from a navigation system. When combined with a conventional series HEV powertrain optimal EM control strategy, in which the driving speed profile is given as an input, it results in a novel suitably decoupled and computationally efficient two-step optimal control strategy.

The proposed strategy is shown to outperform in various aspects existing vehicle speed and powertrain EM cooptimization schemes, for an exemplary straight road mission. In particular, as compared to a recently introduced speed and EM split optimization benchmark method it saves $9 \%$ of fuel, while only increasing marginally the computation time. Additionally, as compared to the benchmark of jointly optimizing speed and EM, which is subject to high computational complexity, it produces comparable fuel consumption but with significantly reduced computation time. For a more realistic rural route driving mission, which is also investigated, the proposed two-step optimization method is found to outperform the split optimization benchmark method by $6 \%$. Although the fuel economy is $4.95 \%$ worse than the joint optimization benchmark solution in this case, the two-step approach is much more computationally efficient. Thus, it enables to tackle more 


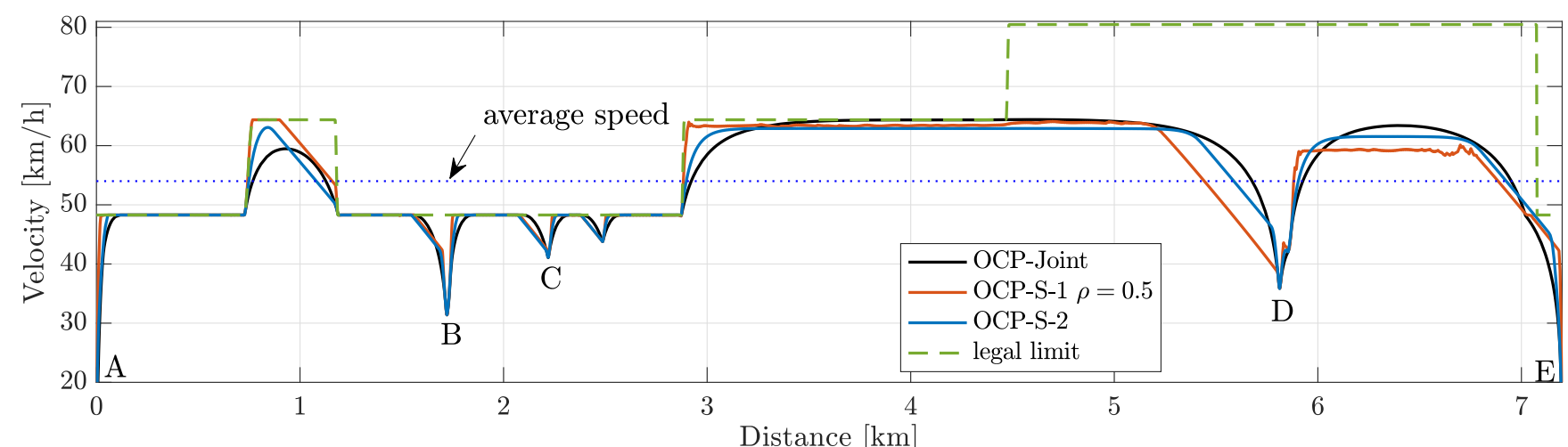

Fig. 9. Optimized speed profiles with respect to traveled distance for the $7.2 \mathrm{~km}$ rural route. The markers A, B, C, D, E correspond to the intersections shown in Fig. 8.
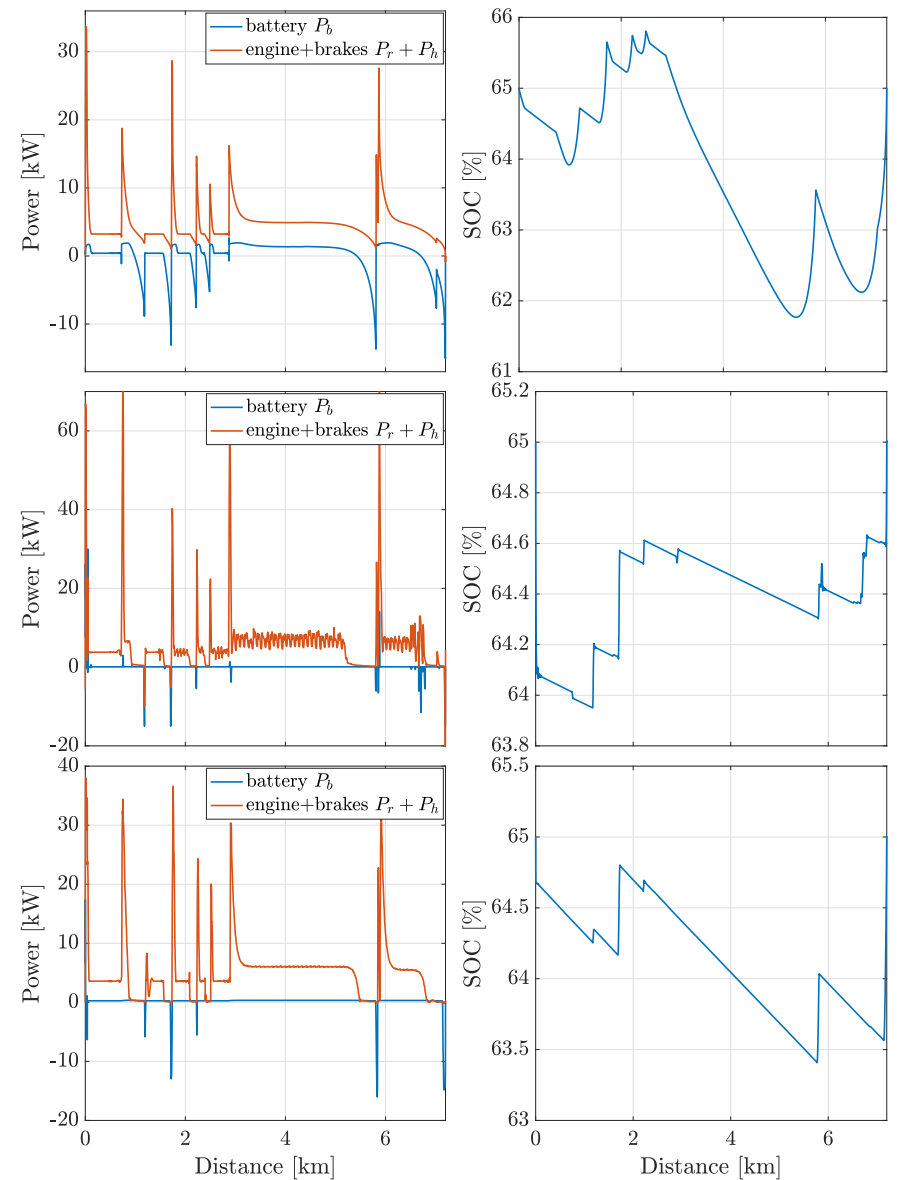

Fig. 10. Power flow and battery SOC profiles solved by the OCP-Joint (top), the OCP-Split-1 [36] for $\rho=0.5$ (middle) and the proposed OCP-Split-2 (bottom) for the $7.2 \mathrm{~km}$ rural road.

complex realistic missions, which the joint scheme cannot feasibly optimize due to its computational complexity.

Future research efforts will be devoted to extending the novel two-step optimization algorithm to further HEV powertrain architectures, beyond the series one, as well as to main powertrain features that are different from the ones in the present study, such as nonlinear fuel consumption map and engine start-stop-system.

\section{REFERENCES}

[1] A. Sciarretta, G. D. Nunzio, and L. L. Ojeda, "Optimal ecodriving control: Energy-efficient driving of road vehicles as an optimal control problem," IEEE Control Systems Magazine, vol. 35, no. 5, pp. 71-90, 2015.

[2] S. E. Li, H. Peng, K. Li, and J. Wang, "Minimum fuel control strategy in automated car-following scenarios," IEEE Transactions On Vehicular Technology, vol. 61, no. 3, pp. 998-1007, 2012.

[3] J. Han, A. Vahidi, and A. Sciarretta, "Fundamentals of energy efficient driving for combustion engine and electric vehicles: An optimal control perspective," Automatica, no. 103, pp. 558-572, 2019.

[4] J. Lee, "Vehicle inertia impact on fuel consumption of conventional and hybrid electric vehicles using acceleration and coast driving strategy," Ph.D. dissertation, VirginiaTech., USA, 2009.

[5] E. Hellstrom, M. Ivarsson, J. Aslund, and L. Nielsen, "Look-ahead control for heavy trucks to minimize trip time and fuel consumption," Control Engineering Practice, vol. 17, no. 2, pp. 245-254, 2009.

[6] B. Asadi and A. Vahidi, "Predictive cruise control: Utilizing upcoming traffic signal information for improving fuel economy and reducing trip time," IEEE Transactions Control Systems Technology, vol. 19, no. 3, pp. 707-714, 2011.

[7] C. R. He, H. Maurer, and G. Orosz, "Fuel consumption optimization of heavy-duty vehicles with grade, wind, and traffic information," Journal of Computational and Nonlinear Dynamics, vol. 11, no. 6, 2016.

[8] M. A. S. Kamal, M. Mukai, J. Murata, and T. Kawabe, "Model predictive control of vehicles on urban roads for improved fuel economy," IEEE Transactions Control Systems Technology, vol. 21, no. 3, pp. 831-841, 2012.

[9] A. A. Malikopoulos, C. G. Cassandras, and Y. J. Zhang, "A decentralized energy-optimal control framework for connected automated vehicles at signal-free intersections," Automatica, vol. 93, pp. 244-256, 2018.

[10] E. Ozatay, S. Onori, J. Wollaeger, U. Ozguner, G. Rizzoni, D. Filev, J. Michelini, and S. D. Cairano, "Cloud-based velocity profile optimization for everyday driving: A dynamic-programming-based solution," IEEE Transactions on Intelligent Transportation Systems, vol. 15, no. 6, pp. 2491-2505, 2014.

[11] G. Ma, M. Ghasemi, and X. Song, "Integrated powertrain energy management and vehicle coordination for multiple connected hybrid electric vehicles," IEEE Transactions On Vehicular Technology, vol. 67, no. 4, pp. 2893-2899, 2018.

[12] V. Turri, B. Besselink, and K. H. Johansson, "Cooperative look-ahead control for fuel efficient and safe heavy-duty vehicle platooning," IEEE Transactions on Control Systems Technology, vol. 25, no. 1, pp. 12-28, 2017.

[13] W. Dib, L. Serrao, and A. Sciarretta, "Optimal control to minimize trip time and energy consumption in electric vehicles," 2011, pp. 1-8.

[14] A. A. Malikopoulos, "Supervisory power management control algorithms for hybrid electric vehicles: A survey," IEEE Transactions on Intelligent Transportation Systems, vol. 15, no. 5, pp. 1869-1885, 2014.

[15] S. G. Wirasingha and A. Emadi, "Classification and review of control strategies for plug-in hybrid electric vehicles," IEEE Transactions On Vehicular Technology, vol. 60, no. 1, pp. 111-122, 2011.

[16] C. M. Martinez, X. Hu, D. Cao, E. Velenis, B. Gao, and M. Wellers, "Energy management in plug-in hybrid electric vehicles: Recent progress 
and a connected vehicles perspective," IEEE Transactions On Vehicular Technology, vol. 66, no. 6, pp. 4534-4549, 2017.

[17] W. Zhou, C. Zhang, J. Li, and H. K. Fathy, "A pseudospectral strategy for optimal power management in series hybrid electric powertrains," IEEE Transactions On Vehicular Technology, vol. 65, no. 6, pp. 48134825, 2016.

[18] J. Liu, Y. Chen, J. Zhan, and F. Shang, "Heuristic dynamic programming based online energy management strategy for plug-in hybrid electric vehicles," IEEE Transactions On Vehicular Technology, vol. 68, no. 5, pp. 4479-4493, 2019.

[19] A. Sciarretta, M. Back, and L. Guzzella, "Optimal control of parallel hybrid electric vehicles," IEEE Transactions on Control Systems Technology, vol. 12, no. 3, pp. 352-363, 2004.

[20] L. Serrao, S. Onori, and G. Rizzoni, "A comparative analysis of energy management strategies for hybrid electric vehicles," Journal of Dynamic Systems, Measurement, and Control, vol. 133, 2011.

[21] F. Yan, J. Wang, and K. Huang, "Hybrid electric vehicle model predictive control torque-split strategy incorporating engine transient characteristics," IEEE Transactions On Vehicular Technology, vol. 61, no. 6, pp. 2458-2467, 2012.

[22] J. Zhang and T. Shen, "Real-time fuel economy optimization with nonlinear mpc for phevs," IEEE Transactions On Control Systems Technology, vol. 24, no. 6, pp. 2167-2175, 2016.

[23] Y. Zhang, H. Liu, and Q. Guo, "Varying-domain optimal management strategy for parallel hybrid electric vehicles," IEEE Transactions On Vehicular Technology, vol. 63, no. 2, pp. 603-616, 2014.

[24] T. Liu, X. Hu, S. E. Li, and D. Cao, "Reinforcement learning optimized look-ahead energy management of a parallel hybrid electric vehicle," IEEE Transactions on Mechatronics, vol. 22, no. 4, pp. 1497-1507, 2017.

[25] Y. L. Murphey, J. Park, Z. Chen, M. L. Kuang, M. A. Masrur, and A. M. Phillips, "Intelligent hybrid vehicle power control — part i: Machine learning of optimal vehicle power," IEEE Transactions On Vehicular Technology, vol. 61, no. 8, pp. 3519-3530, 2012.

[26] Y. L. Murphey, J. Park, L. Kiliaris, M. L. Kuang, M. A. Masrur, A. M Phillips, and Q. Wang, "Intelligent hybrid vehicle power control part ii: Online intelligent energy management," IEEE Transactions On Vehicular Technology, vol. 62, no. 1, pp. 69-79, 2013.

[27] L. Liang, Z. Liyan, Y. Chao, X. Rui, Y. Sixiong, and H. Zongqi, "A novel combinatorial optimization algorithm for energy management strategy of plug-in hybrid electric vehicle," Journal of the Franklin Institute, vol. 354, no. 15, pp. 6588-6609, 2017.

[28] A. Sciarretta and L. Guzzella, "Control of hybrid electric vehicles," IEEE Transactions on Control Systems Technology, vol. 27, no. 2, pp. 60-70, 2007.

[29] S. Stockar, V. Marano, M. Canova, G. Rizzoni, and L. Guzzella, "Energy-optimal control of plug-in hybrid electric vehicles for realworld driving cycles," IEEE Transactions On Vehicular Technology, vol. 60, no. 7, pp. 2949-2962, 2011.

[30] W. Shabbir and S. A. Evangelou, "Exclusive operation strategy for the supervisory control of series hybrid electric vehicles," IEEE Transactions On Control Systems Technology, vol. 24, no. 6, pp. 2190-2198, 2016.

[31] _ - "Threshold-changing control strategy for series hybrid electric vehicles," Applied Energy, pp. 761-775, 2019.

[32] R. Lot and S. A. Evangelou, "Green driving optimization of a series hybrid electric vehicle," in 52nd IEEE Conference on Decision and Control, 2013, pp. 2200-2207.

[33] S. Uebel, N. Murgovski, C. Tempelhahn, and B. Baker, "Optimal energy management and velocity control of hybrid electric vehicles," IEEE Transactions On Vehicular Technology, vol. 67, no. 1, pp. 327-337, 2018.

[34] B. Chen, S. A. Evangelou, and R. Lot, "Series hybrid electric vehicle simultaneous energy management and driving speed optimization," IEEE/ASME Transactions on Mechatronics, p. doi: 10.1109/TMECH.2019.2943320, 2019.

[35] S. Uebel, N. Murgovski, C. Tempelhahn, and B. Baker, "A two-level mpc for energy management including velocity control of hybrid electric vehicles," IEEE Transactions On Vehicular Technology, vol. 68, no. 6, pp. 5494-5505, 2019.

[36] B. Chen, S. A. Evangelou, and R. Lot, "Fuel efficiency optimization methodologies for series hybrid electric vehicles," in IEEE Vehicle Power and Propulsion Conference (VPPC), 2018.

[37] T. Markel, A. Brooker, T. Hendricks, V. Johnson, K. Kelly, B. Kramer, M. O'Keefe, S. Sprik, and K. Wipke, "Advisor: a systems analysis tool for advanced vehicle modeling," Journal of Power Sources, vol. 110, no. 2, pp. 255-266, 2002.
[38] V. Johnson, "Battery performance models in advisor," Journal of Power Source, vol. 110, no. 2, pp. 321-329, 2002.

[39] F. Biral, M. Da Lio, and E. Bertolazzi, "Combining safety margins and user preferences into a driving criterion for optimal control-based computation of reference maneuvers for an adas of the next generation," IEEE Intelligent Vehicles Symposium, Proceedings, vol. 2005, pp. 3641, 2005.

[40] W. Shabbir and S. A. Evangelou, "Real-time control strategy to maximize hybrid electric vehicle powertrain efficiency," Applied Energy, pp. 512-522, 2014.

[41] J. M. Lujan, C. Guardiola, B. Pla, and A. Reig, "Analytical optimal solution to the energy management problem in series hybrid electric vehicles," IEEE Transactions on Vehicular Technology, vol. 67, no. 8, pp. 6803-6813, 2018

[42] A. V. Rao, D. Benson, C. Darby, M. Patterson, C. Francolin, I. Sanders, and G. Huntington, "Gpops: A matlab software for solving multiplephase optimal control problems using the gauss pseudospectral method," ACM Transactions on Mathematical Software, vol. 37, no. 2, 2010.

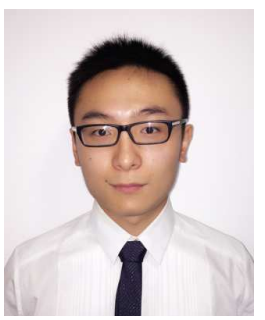

Boli Chen (M'16) received the B. Eng. in Electrical and Electronic Engineering in 2010 from Northumbria University, UK. In 2011 and 2015, he respectively received the $\mathrm{MSc}$ and the $\mathrm{Ph} . \mathrm{D}$. in Control Systems from Imperial College London, UK. Currently, he is a Lecturer in the Department of Electronic and Electrical Engineering, University College London, U.K. His research focuses on control, optimization, estimation and identification of a range of complex dynamical systems, mainly from automotive and power electronics areas.

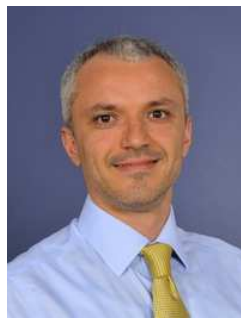

Simos A. Evangelou (M'05-SM'17) received the B.A./M.Eng. degree in electrical and information sciences from the University of Cambridge, Cambridge, U.K. in 1999, and the Ph.D. degree in control engineering from Imperial College London, London, U.K. in 2004. He is currently a Reader with the Department of Electrical and Electronic Engineering, Imperial College London, London, U.K. Dr. Evangelou is a Member of IFAC Technical Committee Automotive Control and on the editorial board of international journals and conferences, including the IEEE Control Systems Society Conference Editorial Board.

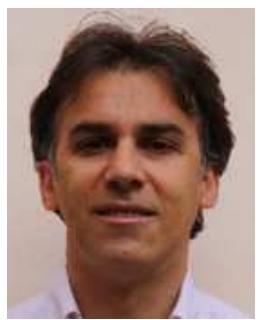

Roberto Lot is Professor of Automotive Engineering at the University of Southampton (UK) since 2014. He received a $\mathrm{PhD}$ in Mechanics of Machines in 1998 and a Master Degree cum laude in Mechanical Engineering in 1994 from the University of Padova (Italy). His research interests include dynamics and control of road and race vehicles, contributing to make our vehicles safer, faster, and eco-friendlier. He has directed several national and international research projects and published more than 100 scientific papers. 